\title{
Aprendizaje mediante videotutoriales en estudiantes de nivel universitario - 2019
}

Learning through video tutorials in university level students - 2019

Aprendizagem por meio de tutoriais em vídeo em estudantes de nível universitário - 2019

\section{ARTÍCULO GENERAL}

Edgardo Félix Palomino Torres

edgardo.palomino@unh.edu.pe https://orcid.org/0000-0002-4252-0704

Universidad Nacional de Huancavelica, Huancavelica - Perú

\author{
Edgar Augusto Salinas Loarte \\ edgar.salinas@unh.edu.pe \\ https://orcid.org/0000-0003-4081-3834 \\ Universidad Nacional de Huancavelica, \\ Huancavelica - Perú
}

Yuri Sánchez Solís yuri.sanchez@unh.edu.pe https://orcid.org/0000-0003-1300-728X Universidad Nacional de Huancavelica, Huancavelica - Perú

Recibido 16 de Diciembre 2020 | Arbitrado y aceptado 06 de Enero 2021 | Publicado el 01 de Marzo 2021

\section{RESUMEN}

El objetivo general de esta investigación es demostrar que la aplicación del video tutorial mejora el aprendizaje del nivel universitario 2019. La metodología utilizada se basó en un diseño experimental, de tipo cuasiexperimental, con un nivel pretest y postest con dos grupos de estudio. Como conclusión se puede mencionar que luego del análisis de las pruebas escritas realizadas tanto al grupo experimental como al grupo de control, se aprecia que la aplicación de video tutoriales mejora el aprendizaje conceptual, actitudinal y procedimental de los estudiantes del grupo experimental, de la misma manera se logró comprobar que la hipótesis general planteada afirma que, la aplicación del videotutorial mejora significativamente el aprendizaje de estudiantes de administración de la Facultad de Ciencias Empresariales de la Universidad Nacional de Huancavelica, Perú-2019, la cual se comprobó mediante el uso de la prueba U de Mann-Whitney, con un margen de error del $5 \%, \alpha=0,05$.

Palabras claves: aprendizaje, actitudinal, conceptual, procedimental, videotutoriales

\section{ABSTRACT}

The general objective of this research is to demonstrate that the application of the video tutorial improves learning at the university level - 2019. The methodology used was based on an experimental design, quasiexperimental, with a pretest and posttest level with two study groups. In conclusion, it can be mentioned that after the analysis of the written tests carried out both in the experimental group and in the control group, it can be seen that the application of video tutorials improves the conceptual, attitudinal and procedural learning of the students of the experimental group, in the same way. In this way, it was possible to verify that the general hypothesis stated affirms that the application of the video tutorial significantly improves the learning of administration students of the Faculty of Business Sciences of the National University of Huancavelica, Peru-2019, which was verified through the use of the Mann-Whitney U test, with a margin of error of $5 \%, \alpha=0.05$.

Keywords: learning, attitudinal, conceptual, procedural, video tutorials

\section{RESUMO}

O objetivo geral desta pesquisa é demonstrar que a aplicação do vídeo tutorial melhora o aprendizado no nível universitário - 2019. A metodologia utilizada foi baseada em um design experimental, quaseexperimental, com um nível de préteste e pós-teste com dois grupos de estudo. Em conclusão, pode-se mencionar que após a análise dos testes escritos realizados tanto no grupo experimental quanto no grupo controle, pode-se constatar que a aplicação de videotutoriais melhora o aprendizado conceitual, atitudinal e processual dos alunos de o grupo experimental, da mesma forma. Desta forma, foi possível verificar que a hipótese geral afirmada afirma que a aplicação do vídeo tutorial melhora significativamente o aprendizado dos alunos de administração da Faculdade de Ciências Empresariais da Universidade Nacional de Huancavelica , Peru-2019, que foi verificado por meio do teste MannWhitney U, com margem de erro de $5 \%, \alpha=0,05$.

Palavras-chave: aprendizagem, atitudinal, conceitual, procedimental, tutoriais em vídeo 


\section{Introducción}

A comienzos y mediados de los ochenta, la integración de estas tecnologías en las escuelas comienzan a ser un tema muy estudiado por lo que se sientan las bases de lo que más tarde conoceríamos como el internet o World Wide Web, (Macau, 2004) cuyo auge se desarrollaría totalmente en la década de los años 90 como el internet o red mundial de información, en consecuencia, el internet acelera la comunicación y el flujo de la información por lo que el termino nuevas tecnologías se transformaría en tecnologías de la información y la comunicación.

Las TIC propician nuevas formas de aprender que, por supuesto, no sustituyen a las tradicionales, lo que hacen es ampliar y enriquecer las posibilidades de educación. Lo nuevo está en la forma en que usamos los recursos, tanto los recientes como los que no lo son, en su combinación e integración, en el respeto a su código propio de comunicación y sobre todo en el empleo pedagógico, didáctico, que hacemos de cada uno y de todos, integrados como un sistema. (Ferreiro \& De Napoli, 2008, pág. 337)

A raíz de este aspecto histórico, la llegada de las TICs a la educación envuelve nuevas concepciones que han despertado el interés de organizaciones y personas para la realización de estudios que brinden nuevos conocimientos sobre este campo. Actualmente, la educación enfrenta varios desafíos, entre ellos destaca dar respuesta a los constantes cambios económicos, sociales y culturales que se dan hacia el interior de la sociedad.

La utilización de las TIC se ha convertido en un tema por derecho propio y también han llegado a ser incorporadas en cada disciplina (Rodríguez Izquierdo, 2011), sin embargo, en cierta medida, a nivel educativo específicamente en la educación superior, la tecnología como herramienta pedagógica está infrautilizada y en muchos casos sigue estando sin aprovechar hasta ahora, puesto que se ha visto y utilizado la tecnología como un conjunto de prácticas educativas tradicionales, de aquí la necesidad de repensar cómo se produce la integración de las tecnologías en los procesos de aprendizaje y docencia a nivel universitario. 


\section{Características de las TIC}

Las características de las tecnologías de la Información y la comunicación son numerosas, sin embargo, se representan en la Tabla 1 las más importantes:

\section{Tabla 1}

Características de las TIC

\begin{tabular}{ll}
\hline CARACTERÍSTICAS & DEFINICIÓN \\
\hline Inmaterialidad. & $\begin{array}{l}\text { En líneas generales podemos decir que las TIC realizan la } \\
\text { creación (aunque en algunos casos sin referentes reales, } \\
\text { como pueden ser las simulaciones), el proceso y la } \\
\text { comunicación de la información. }\end{array}$ \\
\hline Interactividad. & $\begin{array}{l}\text { Es posiblemente la característica más importante de las TIC } \\
\text { para su aplicación en el campo educativo. Mediante las TIC } \\
\text { se consigue un intercambio de información entre el usuario y } \\
\text { el ordenador. }\end{array}$ \\
\hline Interconexión & $\begin{array}{l}\text { Hace referencia a la creación de nuevas posibilidades } \\
\text { tecnológicas a partir de la conexión entre dos tecnologías. }\end{array}$ \\
\hline Instantaneidad & $\begin{array}{l}\text { Las redes de comunicación y su integración con la } \\
\text { informática, han posibilitado el uso de servicios que } \\
\text { permiten la comunicación y transmisión de la información, } \\
\text { entre lugares alejados físicamente, de una forma rápida. }\end{array}$ \\
\hline Su objetivo es que la información de distinto tipo (sonidos, \\
texto, imágenes, animaciones, etc.) pueda ser transmitida por \\
los mismos medios al estar representada en un formato único \\
universal.
\end{tabular}

Fuente: (Ortí, 2011)

En la misma manera estas características, hacen que las transformaciones sociales, culturales y económicas que enmarcan la sociedad del siglo XXI sean abrumadoras. Igualmente su uso en los procesos de enseñanza y aprendizaje ya sea presencial o a 
distancia, en forma uni o bidireccionalmente, permiten el intercambio de roles y mensajes, (Castro, Guzmán, \& Casado, 2007, pág. 224), en este sentido las TIC permiten que fluya el proceso de comunicación entre estudiantes, estudiantes - docentes y estudiantes materiales, individuos que consumen, procesan y entregan información, la cual puede ser utilizada en tiempo real o almacenada para tener acceso a ella cuando sea necesario.

\section{Informática y aprendizaje}

Como lo indica, (Villarroel, 2004), la informática y el proceso de aprendizaje se refiere al fenómeno de la inclusión de las nuevas tecnologías en la formación educativa, y se le denomina como "La revolución digital de la educación", esto exige al sujeto utilizar sistemas altamente tecnificados y demandantes de alto flujo de información, que obtiene por medio de las nuevas tecnologías de la información.

Un tutor no debería proveer la inteligencia para lograr el aprendizaje, no debería realizar la planeación y el monitoreo del progreso de los estudiantes, porque estas son las actividades que los estudiantes deberían ejecutar ellos mismos para aprender. Lo que un tutor debería hacer es apoyarlos temporalmente para permitir que los aprendices ejecuten a un nivel justo y más allá de su nivel corriente de habilidad. (Scardamalia, Bereiter, Mclean, Swallow, \& \&Woodruff, 1989, pág. 245)

\section{Aprendizaje a distancia (e - learning)}

El e-learning está creciendo en importancia en todo el mundo. Las instituciones educativas y muchas empresas incorporan plataformas de e-learning en sus procesos formativos sin una evaluación en profundidad de las alternativas disponibles. (Adam, Vallés, \& Rodríguez, 2001, pág. 3), lo que permite definir al e-learning como un sistema de enseñanza y aprendizaje basado en el uso de las TIC, lo que permite la continuación de las clases sin las restricciones tradicionales de espacio, tiempo.

Existen unas series de ventajas y desventajas de este tipo de aprendizaje con respecto a la educación tradicional que se explicaran en la Tabla 2 


\section{Tabla 2}

Ventajas y desventajas del e-learning con respecto al aprendizaje tradicional

\begin{tabular}{|c|c|c|}
\hline \multirow{6}{*}{$\frac{\stackrel{\frac{\pi}{\pi}}{\frac{\pi}{\pi}}}{\underbrace{0}_{0}}$} & APRENDIZAJE TRADICIONAL & E-LEARNING \\
\hline & Feedback inmediato Es familiar & Centrado en el alumno y a su propio ritmo \\
\hline & tanto a los estudiantes como a los & Tiempo y localización flexible \\
\hline & profesores Motiva a los & Eficaz en coste para el alumno \\
\hline & estudiantes Cultivo de una & Potencialmente disponible para una \\
\hline & comunidad social & $\begin{array}{l}\text { audiencia global - Acceso ilimitado al } \\
\text { conocimiento }\end{array}$ \\
\hline \multirow{6}{*}{  } & Centrado en el instructor & Falta de retorno inmediato en el e-learning \\
\hline & Restricción de tiempo y lugar & asíncrono \\
\hline & Más caro de comunicar & Aumenta el tiempo de preparación para el \\
\hline & & profesor y el coste \\
\hline & & No es cómodo para algunos \\
\hline & & $\begin{array}{l}\text { Potencialmente introduce más frustración, } \\
\text { ansiedad, y confusión }\end{array}$ \\
\hline
\end{tabular}

Fuente: (Adam, et al., 2001)

Software educativo.

Los softwares educativos como lo indica (Gros, 1997), está sistematizado en base a cuatro categorías que se describen a continuación: propone la siguiente sistematización en base a cuatro categorías:

-Tutorial: enseña un determinado contenido.

-Práctica y ejercitación: La repetición de una determinada tarea, en el desarrollo de un tema, contribuye en adquirir destreza.

-Simulación: proporciona entornos de aprendizaje similares a situaciones reales.

-Hipertexto e hipermedia: Entorno de aprendizaje no lineal.

\section{Teoría del aprendizaje significativo}

La teoría del aprendizaje son un conjunto global de fundamentos, hechos, enfoques y perspectivas teóricas que pretenden exponer explicaciones más o menos generales de los elementos o factores involucrados en el proceso de cambio que las personas experimentan, (Coll, 2000), esto como resultado de su experiencia y de su relación con el ambiente que lo rodea. 


\section{Aprendizaje de contenidos conceptuales}

Los conceptos se refieren a un conjunto de hechos, objetos o símbolos que tienen características comunes; y los principios, a los cambios en los hechos, objetos o situaciones en relación con otros (Coll, 2000), de esta manera se puede mencionar que para ambos casos, su aprendizaje necesita comprender de qué se trata, cuál es su significado, pues el propósito es aprender a interpretar su significado y no tener un aprendizaje literal de las cosas. Es por eso que, aprender conceptos y principios esto da una transformación de las estructuras mentales. Esto involucra una construcción personal, una reestructuración del conocimiento previo, con la finalidad de generar nuevas estructuras conceptuales que permitan combinar estos conocimientos como los anteriores, mediante procesos de reflexión y toma de conciencia conceptual.

\section{Aprendizaje de contenidos procedimentales}

Los aprendizajes procedimentales destinan un conjunto de acciones, de formas de actuar a fin de lograr metas. Se trata de unos conocimientos con los cuales nos referimos al saber hacer y su aprendizaje supone, en último término, que se sabrá usar y aplicar en otras situaciones de persecución de metas. En ellos agrupamos las habilidades y capacidades básicas para actuar de alguna manera, a las estrategias que uno aprende para solucionar problemas o a las técnicas y actividades sistematizadas relacionadas con aprendizajes concretos.

Se aprende en definitiva cuando se adquieren los procedimientos, es una vía, un camino, un recurso para llegar a objetivos con la particularidad de que lo más interesante del aprendizaje es que se trata de adquirir una secuencia de pasos o componentes, (Valls, 1995), de allí que hablar de enseñar y aprender contenidos procedimentales quiere decir que insistimos en una determinada orden de actuar hacia una meta

\section{Aprendizaje de contenidos actitudinales}

En vista de la diversidad de individuos que pueden estar presentes en una misma área de clases, cada uno con características, habilidades y destrezas diferentes es necesario que se tomen nuevas actitudes, para que cada uno de los individuos puedan adaptarse y desarrollar sus conocimientos aprovechando sus propios recursos intelectuales. 
La enseñanza-aprendizaje de actitudes y valores interculturales tienen un fuerte impacto social, pues permite desarrollar predisposiciones a actuar de forma respetuosa ante una sociedad en constante y vertiginoso cambio cultural, donde los conocimientos y las conductas concretas quedan rápidamente caducos. La multiculturalidad nos exige el reto educativo de encontrar los valores mínimos, consensuados, que permitan el diálogo y la igualdad entre seres humanos y el cambio de actitudes que favorezca el respeto a la diferencia y la diversidad cultural y promueva la convivencia pacífica entre los diferentes (García López, 2003, pág. 55)

\section{Video tutoriales para el aprendizaje}

Actualmente en la era de la tecnología el uso de videos tutoriales para adquirir algún tipo de aprendizaje no es algo fuera de orden, de hecho, los jóvenes en edades para ingresar a la universidad son asiduos al uso de la tecnología, acostumbrados a buscar por la internet todo los que le genere curiosidad para poder adquirir algún conocimiento.

Ahora la idea es convertir esos videos adaptados a los contenidos de las asignaturas y poder impartirles los conocimientos a través de diversas plataformas, para ello es necesario tomar en cuenta ciertos aspectos como lo indica (Bengochea \& Medina, 2013, pág. 84), los cuales se expresan a continuación:

- Planificarlos con una duración de entre 10 y 15 minutos. En caso de necesitar más tiempo, repartirlo entre varios videos.

- Elaborar un script de la secuencia de imágenes, el texto, la voz y los gestos del presentador antes de empezar a grabar.

- Elegir el formato más apropiado para el contenido que se va a tratar: busto parlante, diapositivas, animaciones, acciones en pantalla, etc. o una combinación de todos ellos.

- Describir al comienzo lo que se va a aprender en el video.

- Conectar cada pieza nueva de información a la información previa.

- Sincronizar las imágenes, el texto y el sonido.

- Usar un lenguaje sencillo y claro y una voz activa, si es posible. 
Entre los materiales multimedia, el video tutorial se presenta como una herramienta que se erige como un recurso didáctico apropiado para mejorar la absorción de conocimientos, el aprendizaje percibido por el alumno y crear sinergias con otros recursos y métodos de enseñanza (Jiménez Castillo \& Marín Carrillo, 2012, pág. 69)

Para finalizar se expresa que el objetivo general de esta investigación es demostrar que la aplicación del video tutorial mejora el aprendizaje del nivel universitario - 2019 . La metodología utilizada se basó en un diseño experimental, de tipo cuasiexperimental, con un nivel pretest y postest con dos grupos de estudio.

\section{Metodología}

La metodología aplicada para esta investigación es de diseño experimental. Este diseño es aquel según el cual el investigador manipula una variable experimental no comprobada, bajo condiciones estrictamente controlada. (Palella Stracuzzi \& Martins Pestana, 2010). A través de este diseño se describe de qué modo y por qué causa se produce o puede producirse un fenómeno.

Los objetivos específicos de esta investigación se basaron en demostrar que la aplicación del video tutorial mejora el aprendizaje conceptual en estudiantes de nivel universitario, demostrar que la aplicación del video tutorial mejora el aprendizaje procedimental en estudiantes de nivel universitario, y demostrar que la aplicación del video tutorial mejora el aprendizaje actitudinal de los estudiantes a nivel universitario.

El tipo de investigación a realizar es cuasiexperimental, este se usa cuando no es factible utilizar un diseño experimental verdadero, es un método de control parcial, (Palella Stracuzzi \& Martins Pestana, 2010), se basa en la identificación de los factores que puedan intervenir en la validez interna y externa del mismo. Los datos extraídos indagan en la relación entre las variables de estudio: aplicación de video tutoriales, y el aprendizaje de los estudiantes.

De la misma manera el nivel de esta investigación utilizado es el pretest y post con dos grupos, que consiste en aplicar de manera simultánea un pretest a dos grupos similares. (Palella Stracuzzi \& Martins Pestana, 2010), así posteriormente un grupo recibe el tratamiento experimental y el otro grupo es de control. Pues demostrar la aplicación de video tutoriales puede determinar el aprendizaje de los estudiantes. 


\section{Población y muestra}

La medida de la población y muestra que será estudiada, es una parte importante en el desarrollo de una investigación. La población es el conjunto de unidades de las que se desea obtener información y sobre las que se van a generar conclusiones, en otra forma, es el conjunto finito o infinito de elementos, personas o cosas pertenecientes a la investigación (Palella Stracuzzi \& Martins Pestana, 2010)

La población de esta investigación está constituida por 236 estudiantes específicamente de Administración de la Facultad de Ciencias Empresariales de la Universidad Nacional de Huancavelica, Perú. La muestra tomada fue de 46 estudiantes divididos en dos grupos de 23 estudiantes cada uno estructurados en un grupo experimental a los que se les aplicaran los video tutoriales y un grupo de control, que seguirá con su aprendizaje tradicional.

\section{Técnicas de recolección de datos:}

Para esta investigación se procedió a utilizar tres test a ambos grupos de estudio como técnica principal de recolección de datos. El test tiene como objeto lograr información sobre rasgos definidos de la personalidad, la conducta o determinados comportamientos y características individuales o colectivas de la persona (inteligencia, actitudes, aptitudes, rendimiento entre otros), (Palella Stracuzzi \& Martins Pestana, 2010)

Los test aplicados son los siguientes:

-1era Prueba escrita: Test de conocimientos conceptuales

-2da Prueba escrita: Test de conocimientos procedimentales

-3era Prueba escrita: Test de conocimientos actitudinales

La 1era prueba escrita consta 10 preguntas de selección simple, para determinar el nivel de aprendizaje del conocimiento conceptual de los estudiantes. El instrumento se puede detallar en la Tabla 3.

\section{Tabla 3}

Instrumento de recolección de datos para determinar el nivel de aprendizaje del conocimiento conceptual de los estudiantes de administración de la Facultad de Ciencias Empresariales de la Universidad Nacional de Huancavelica, Perú-2019 


\begin{tabular}{|c|c|c|c|}
\hline & PREGUNTAS & \multicolumn{2}{|c|}{ OPCIONES DE RESPUESTA } \\
\hline 1 & $\begin{array}{l}\text { Una sociedad políticamente } \\
\text { organizada que ejerce Poder } \\
\text { Soberano en un territorio } \\
\text { determinado, es lo que } \\
\text { denominamos: }\end{array}$ & $\begin{array}{l}\text { a) Estado } \\
\text { b) Comunidad }\end{array}$ & $\begin{array}{l}\text { c) País } \\
\text { d) Población }\end{array}$ \\
\hline 2 & El estado está compuesto por: & $\begin{array}{l}\text { a) Población, territorio } \\
\text { y país } \\
\text { b) Sociedad, escudo y } \\
\text { bandera }\end{array}$ & $\begin{array}{l}\text { c) Sociedad, territorio, } \\
\text { escudo y bandera } \\
\text { d)Población, territorio y } \\
\text { poder }\end{array}$ \\
\hline 3 & $\begin{array}{l}\text { Dos ejemplos de Organismos } \\
\text { Constitucionalmente } \\
\text { Autónomos son: }\end{array}$ & $\begin{array}{l}\text { a) Ministerio Publico e } \\
\text { INDECOPI } \\
\text { b) RENIEC Y } \\
\text { Ministerio Publico }\end{array}$ & $\begin{array}{l}\text { c)RENIEC y Sedapal } \\
\text { d)INDECOPI y Sedapal }\end{array}$ \\
\hline 4 & $\begin{array}{l}\text { Un ejemplo de una entidad de } \\
\text { prestación de servicios } \\
\text { públicos es: }\end{array}$ & $\begin{array}{l}\text { a)OSIPTEL } \\
\text { b)INDECOPI }\end{array}$ & $\begin{array}{l}\text { c)SBS } \\
\text { d)EsSalud }\end{array}$ \\
\hline 5 & $\begin{array}{l}\text { ¿De qué se encarga el Poder } \\
\text { Legislativo? }\end{array}$ & $\begin{array}{l}\text { a)De hacer que se } \\
\text { cumpla las leyes } \\
\text { b)De ejecutar las leyes }\end{array}$ & $\begin{array}{l}\text { c)De Gobernar } \\
\text { d)De aprobar, modificar } \\
\text { o derogar las leyes }\end{array}$ \\
\hline 6 & $\begin{array}{l}\text { ¿Quién es el jefe supremo de } \\
\text { las fuerzas armadas y de la } \\
\text { policía nacional de Perú? }\end{array}$ & $\begin{array}{l}\text { a)El ministro de } \\
\text { defensa } \\
\text { b)El presidente de la } \\
\text { república }\end{array}$ & $\begin{array}{l}\text { c)El ministerio del } \\
\text { interior } \\
\text { d)El presidente del } \\
\text { congreso }\end{array}$ \\
\hline 7 & $\begin{array}{l}\text { La corte suprema de justicia } \\
\text { forma parte }\end{array}$ & $\begin{array}{l}\text { a)Del poder legislativo } \\
\text { b)Del poder ejecutivo }\end{array}$ & $\begin{array}{l}\text { c)Del poder judicial } \\
\text { d)De los organismos } \\
\text { Constitucionalmente } \\
\text { Autónomos }\end{array}$ \\
\hline 8 & $\begin{array}{l}\text { ¿Quién preside el Consejo de } \\
\text { Ministros? }\end{array}$ & $\begin{array}{l}\text { a)El ministro que sea } \\
\text { designado } \\
\text { b)El ministro de } \\
\text { defensa }\end{array}$ & $\begin{array}{l}\text { c)El presidente de la } \\
\text { república } \\
\text { d)El presidente de la } \\
\text { Corte Suprema de } \\
\text { Justicia }\end{array}$ \\
\hline 9 & $\begin{array}{l}\text { ¿Cuáles son los roles que el } \\
\text { Estado asume frente a la } \\
\text { ciudadanía? }\end{array}$ & $\begin{array}{l}\text { a)Normativo y } \\
\text { administrativo } \\
\text { b)Regulador y } \\
\text { prestacional }\end{array}$ & $\begin{array}{l}\text { c)Administrativo y } \\
\text { regulador } \\
\text { d)Funcional y normativo }\end{array}$ \\
\hline 10 & $\begin{array}{l}\text { El estado presta servicios a } \\
\text { través de: }\end{array}$ & $\begin{array}{l}\text { a)Entidades Publicas } \\
\text { b)Sociedad Civil }\end{array}$ & $\begin{array}{l}\text { c)Entidades Públicas y a } \\
\text { través de personas } \\
\text { jurídicas o naturales } \\
\text { d)Entidades Públicas y } \\
\text { proveedores autorizados }\end{array}$ \\
\hline
\end{tabular}

Seguidamente se discrimina en la Tabla 4 el instrumento utilizado para determinar el nivel de aprendizaje del conocimiento procedimental de los estudiantes, que consta de preguntas de selección simple y de completación. 


\section{Tabla 4}

Instrumento de recolección de datos para determinar el nivel de aprendizaje del conocimiento procedimental de los estudiantes de administración de la Facultad de Ciencias Empresariales de la Universidad Nacional de Huancavelica, Perú-2019

\section{PREGUNTAS}

Relacione los conceptos.

\begin{tabular}{lll}
\hline A & MOF/MPP & $\begin{array}{l}\text { Define la estructura de las entidades, } \\
\text { sus oficinas y las funciones que } \\
\text { corresponden a cada una de ellas. }\end{array}$ \\
\hline B & CAP/PAP/CPE & $\begin{array}{l}\text { Define los puestos, plazas y perfiles de } \\
\text { los recursos humanos necesarios para } \\
\text { que la entidad cumpla su función. }\end{array}$ \\
\hline C & ROF & $\begin{array}{l}\text { Define las funciones de cada uno de } \\
\text { los puestos y plazas para la entidad. }\end{array}$ \\
\hline D & POI & $\begin{array}{l}\text { Establece los objetivos y metas de la } \\
\text { entidad }\end{array}$ \\
\hline E & PEI & $\begin{array}{l}\text { Establece las actividades y proyectos a } \\
\text { través de los cuales se alcanzan las } \\
\text { metas y submetas de la entidad. }\end{array}$ \\
\hline
\end{tabular}

El POI define:

Los Sistemas Administrativos, se aplican a:

Describa la conformación del Poder Legislativo:

$\bar{E} 1$ procedimiento administrativo, en la práctica es llamado:

Los Sistemas Administrativos tienen con meta:

Los Sistemas funcionales son:

Mencione cuales son las características de una regulación de calidad e indica de que se trata cada una de ellas:

Describa el fin del análisis de $\bar{C}$ alidad Regulatoria:

Indica que actividades se llevan a cabo en cada una de las fases para la aplicación del ACR:

De la misma manera, en la Tabla 5 se muestra el instrumento utilizado para determinar el nivel de aprendizaje del conocimiento actitudinal de los estudiantes, y está conformado por preguntas con respuestas abiertas. 


\section{Tabla 5}

Instrumento de recolección de datos para determinar el nivel de aprendizaje del conocimiento actitudinal de los estudiantes de administración de la Facultad de Ciencias Empresariales de la Universidad Nacional de Huancavelica, Perú-2019

\begin{tabular}{l}
\hline PREGUNTAS \\
\hline ¿Trabajas en equipo respetando la opinión de tus compañeros? \\
¿Aceptas sugerencias en el proceso de resolución de problemas? \\
¿Demuestra orden en la presentación de sus trabajos individuales? \\
¿Demuestra orden en la presentación de sus trabajos grupales? \\
¿Demuestra esfuerzo en el logro de sus aprendizajes Conceptuales? \\
¿Demuestra esfuerzo en el logro de sus aprendizajes Procedimentales? \\
¿Has tenido la satisfacción de resolver las actividades planteadas? \\
¿Cuál es tu reacción cuando algo sale mal? \\
¿Cuáles son los aspectos que más valoras cuando trabajas en equipo? \\
¿Conoces tus fortalezas y debilidades?
\end{tabular}

Del mismo modo, en la Tabla 6 se discriminan las categorías utilizadas para poder realizar el análisis estadístico conforme a lo establecido en el reglamento académico vigente de la Universidad, según los siguientes detalles:

\section{Tabla 6.}

Categorías de la condición de los estudiantes

\begin{tabular}{ll}
\hline RANGO DE & CONDICIÓN DEL \\
CALIFICACIÓN & ESTUDIANTE \\
\hline $0-10.4$ & Deficiente \\
\hline $10.5-13.9$ & Regular \\
\hline $14-20$ & Excepcional \\
\hline
\end{tabular}

Igualmente, se expresa que estos instrumentos fueron validados por expertos para comprobar su confiabilidad.

Luego de la recolección de datos y de forma más ampliada, se utilizó para ser procesados utilizando el estadístico IBM SPSS versión 21, sugerido para el uso en estudios desarrollados en las ramas de las ciencias sociales. De la misma manera, para realizar la contrastación de hipótesis se utilizó la prueba U de Mann-Whitney, con un margen de error del $5 \%, \alpha=0,05$. 


\section{Resultados y discusión}

En la tabla 7 se aprecian los resultados correspondientes al test de conocimiento conceptual

Tabla 7

Test de conocimiento Conceptual

\begin{tabular}{lllll}
\hline \multirow{2}{*}{ CONDICIÓN } & \multicolumn{2}{l}{ GRUPO EXPERIMENTAL } & \multicolumn{2}{l}{ GRUPO CONTROL } \\
\cline { 2 - 5 } & FRECUENCIA & PORCENTAJE & FRECUENCIA & PORCENTAJE \\
\hline Excepcional & 17 & $73,91 \%$ & 13 & $56,52 \%$ \\
\hline Regular & 6 & $26,09 \%$ & 10 & $43,48 \%$ \\
\hline Deficiente & 0 & $0 \%$ & 0 & $0 \%$ \\
\hline TOTAL & 23 & $100 \%$ & 23 & $100 \%$ \\
\hline
\end{tabular}

Los estudiantes del grupo experimental tienen mejor nivel de promedio en el aprendizaje conceptual frente a los estudiantes del grupo control. 73,91\% tienen la condición de excepcional, 26,09\% son regulares y el $0 \%$ deficiente. Finalmente se puede notar que no existe estudiante alguno que tiene deficiencias en la resolución del test de conocimiento conceptual aplicado.

Seguidamente en la Tabla 8 se aprecian los resultados arrojados de la aplicación del test de conocimiento procedimental

\section{Tabla 8}

Test de conocimiento Procedimental

\begin{tabular}{lllll}
\hline \multirow{2}{*}{ CONDICIÓN } & \multicolumn{2}{l}{ GRUPO EXPERIMENTAL } & \multicolumn{2}{l}{ GRUPO CONTROL } \\
\cline { 2 - 5 } & FRECUENCIA & PORCENTAJE & FRECUENCIA & PORCENTAJE \\
\hline Excepcional & 18 & $78,26 \%$ & 5 & $21,74 \%$ \\
\hline Regular & 3 & $13,04 \%$ & 17 & $73,91 \%$ \\
\hline Deficiente & 2 & $9 \%$ & 1 & $4 \%$ \\
\hline TOTAL & 23 & $100 \%$ & 23 & $100 \%$ \\
\hline
\end{tabular}

Se observa que gran parte de los estudiantes del grupo experimental, que representan más del 78\% del total, tienen buen promedio en el aprendizaje procedimental a comparación de los estudiantes del grupo control que representan casi el $21 \%$. Asimismo, se puede apreciar que existen estudiantes en ambos grupos que tienen dificultades en el desarrollo del test de conocimiento procedimental. Finalmente, se puede notar que gran cantidad de los estudiantes del grupo control mantiene una condición regular en los resultados de la aplicación del mencionado test. 
En la Tabla 9 se muestran los valores obtenidos de la aplicación del test de conocimiento actitudinal

Tabla 9

Test de conocimiento Actitudinal

\begin{tabular}{lllll}
\hline \multirow{2}{*}{ CONDICIÓN } & \multicolumn{2}{l}{ GRUPO EXPERIMENTAL } & \multicolumn{2}{l}{ GRUPO CONTROL } \\
\cline { 2 - 5 } & FRECUENCIA & PORCENTAJE & FRECUENCIA & PORCENTAJE \\
\hline Excepcional & 17 & $73,91 \%$ & 11 & $47,83 \%$ \\
\hline Regular & 6 & $26,09 \%$ & 11 & $47,83 \%$ \\
\hline Deficiente & 0 & $0 \%$ & 1 & $4 \%$ \\
\hline TOTAL & 23 & $100 \%$ & 23 & $100 \%$ \\
\hline
\end{tabular}

Los resultados acusan claramente que, el aprendizaje actitudinal de los estudiantes del grupo experimental es mayor en comparación de los estudiantes del grupo control, ya que la mayor concentración de estudiantes está en la condición de excepcional, así como se muestra en los resultados observados. Además, solo un estudiante del Grupo Control obtuvo un resultado relativamente deficiente.

\section{Prueba de hipótesis}

\section{Hipótesis General:}

Ho: La aplicación del videotutorial no mejora significativamente el aprendizaje de estudiantes de administración de la Facultad de Ciencias Empresariales de la Universidad Nacional de Huancavelica, Perú-2019

H1: La aplicación del videotutorial mejora significativamente el aprendizaje de estudiantes de administración de la Facultad de Ciencias Empresariales de la Universidad Nacional de Huancavelica, Perú-2019

La prueba de hipótesis general, se realizó a un nivel de significación $=0,05$, en la Tabla10 se muestran las calificaciones generales de los estudiantes.

\section{Tabla 10}

Calificaciones generales de los estudiantes

\begin{tabular}{lll}
\hline N. $^{\mathbf{o}}$ & $\begin{array}{l}\text { Grupo } \\
\text { experimental }\end{array}$ & Grupo control \\
\hline 1 & 16 & 12 \\
\hline 2 & 17 & 13 \\
\hline
\end{tabular}




\begin{tabular}{|c|c|c|}
\hline 3 & 13 & 13 \\
\hline 4 & 17 & 14 \\
\hline 5 & 11 & 12 \\
\hline 6 & 16 & 14 \\
\hline 7 & 17 & 13 \\
\hline 8 & 12 & 12 \\
\hline 9 & 19 & 12 \\
\hline 10 & 13 & 11 \\
\hline 11 & 16 & 14 \\
\hline 12 & 16 & 12 \\
\hline 13 & 16 & 13 \\
\hline 14 & 15 & 12 \\
\hline 15 & 16 & 12 \\
\hline 16 & 17 & 13 \\
\hline 17 & 13 & 13 \\
\hline 18 & 17 & 14 \\
\hline 19 & 11 & 12 \\
\hline 20 & 16 & 14 \\
\hline 21 & 17 & 13 \\
\hline 22 & 13 & 12 \\
\hline 23 & 19 & 12 \\
\hline
\end{tabular}

De la misma manera, en la Tabla 11 se muestra la prueba realizada para la contrastación de hipótesis.

\section{Tabla 11}

Prueba de Mann-Whitney para hipótesis general usando Software estadístico IBM SPSS

\begin{tabular}{ll}
\hline & Calificaciones \\
\hline U de Mann-Whitney & 96,000 \\
$Z$ & $-3,767$ \\
Sig. Asintót. & 0,000165 \\
(bilateral) & \\
\hline
\end{tabular}

Se puede observar en la tabla 12, que la prueba U de Mann-Whitney fue $96.000 \mathrm{y}$ el valor de p (Sig. asintót. (bilateral)) es 0,000165 (menor que 0,05).

Seguidamente, en la Figura 1 se muestra el diagrama de distribución para la toma de decisión de la hipótesis general. 


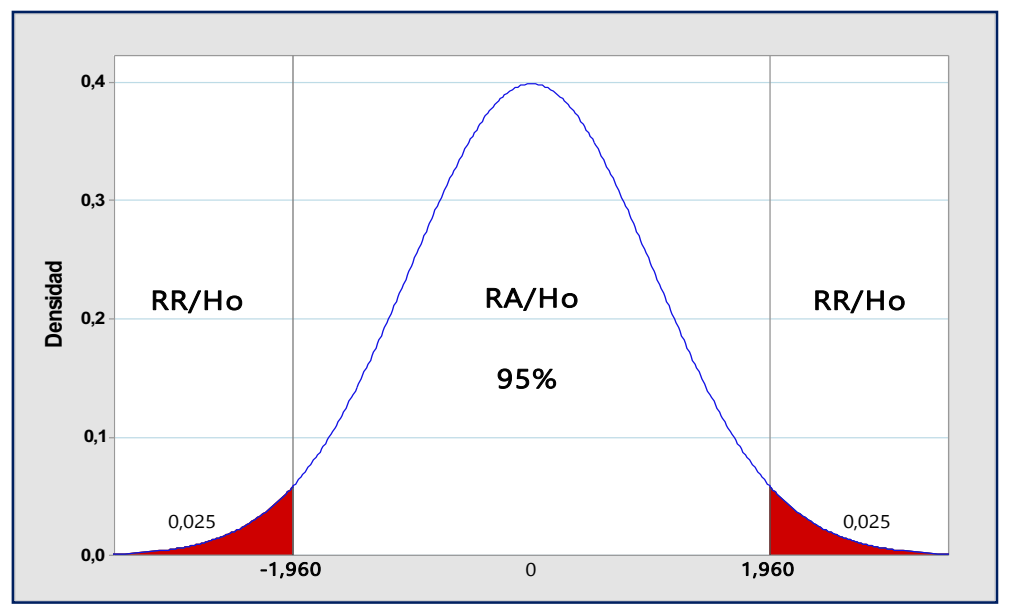

Figura 1 Diagrama de distribución para la toma de decisión de la hipótesis general

En la Figura 1, se percibe que el valor de $Z=-3,767$ y se localiza en la región de rechazo de la hipótesis nula. Esto nos permite concluir que, existe la seguridad para rechazar la hipótesis nula y aceptar la hipótesis alterna, afirmando que, la aplicación del videotutorial mejora significativamente el aprendizaje de estudiantes de administración de la Facultad de Ciencias Empresariales de la Universidad Nacional de Huancavelica, Perú-2019

\section{Referencias bibliográficas}

Adam, M. R., Vallés, R. S., \& Rodríguez, G. I. (2001). E-learning: características y evaluación. Ensayos de economía, Adam, M. R., Vallés, R. S., \& Rodríguez, G. I. M. (2013). E-learning: características y evaluación. Ensayos de economía, 23(43), 143-159.

Bengochea, L., \& Medina, J. A. (2013). El papel de los videotutoriales accesibles en el aprendizaje del futuro. In Actas V Congreso Internacional sobre Aplicación de Tecnologías de la Información y Comunicaciones Avanzadas, (págs. 80-87). Huancayo, Perú.

Castro, S., Guzmán, B., \& Casado, D. (2007). Las Tic en los procesos de enseñanza y aprendizaje. . Laurus, 13(23), 213-234.

Coll, C. (2000). Psicología do ensino. Sau Paulo, Brasil:. Brasil: Editora Artes Médicas Sull. 
Ferreiro, R. F., \& De Napoli, A. (2008). Más allá del salón de clases: Los nuevos ambientes de aprendizajes. Revista complutense de educación, 19(2) , 333-346.

García López, R. (2003). Formación del profesorado en pedagogía intercultural: contenidos actitudinales. Valencia, España: Informe de la Universidad de Valencia.

Gros, B. (1997). Diseños y programas educativos. . Barcelona.: Ariel.

Jiménez Castillo, D., \& Marín Carrillo, G. M. (2012). Asimilación de contenidos y aprendizaje mediante el uso de videotutoriales. Enseñanza \& Teaching 30(2), 6379.

Macau, R. (2004). TIC:¿ para qué?: Funciones de las tecnologías de la información y la comunicación en las organizaciones. . International Journal of Educational Technology in Higher Education (ETHE), 1(1) , 1-12.

Ortí, C. B. (2011). Las tecnologías de la información y comunicación (TIC). . Universidad de Valencia, Unidad de Tecnología Educativa, (951) , 1-7.

Palella Stracuzzi, S., \& Martins Pestana, F. (2010). Metodologia de la Investigación Cuantitativa. Carcas, Venezuela: FEDUPEL, Fondo Editorial de la Universidad Pedagógica Experimental Libertador.

Rodríguez Izquierdo, R. M. (2011). Repensar la relación entre las TIC y la enseñanza. Profesorado 15(1), 9-22.

Scardamalia, M., Bereiter, C., Mclean, R., Swallow, J., \& \&Woodruff, E. (1989). Computer-supported intentional learning environments. . Journal of Educational Computing Research. 5 , 51-68.

Valls, E. (1995). Los Procedimientos. Aprendizaje, enseñanza y evaluación. . Barcelona: Morsor.

Villarroel, E. P. (2004). Educación e Internet.¿ La Próxima Revolución?. Investigaciones en Educación, 4(4), 337-340. 